\title{
Acute kidney injury and stroke: unresolved issues
}

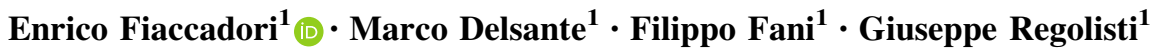

Received: 16 August 2017/Accepted: 18 August 2017/Published online: 1 September 2017

(C) SIMI 2017

Acute kidney injury (AKI) is now considered a major clinical health problem [1, 2]. Its incidence is high, especially among ICU patients, and the syndrome still remains associated with a negative prognosis, both in the short- [3] and in the long-term period [4].

The negative effects of AKI on prognosis appear as a common denominator for this syndrome, independently from the clinical setting being considered [3, 4]. However, some specific subgroups of patients with AKI are characterized by further complexities, as is the case of patients with stroke.

In this regard, a better knowledge of the epidemiology and pathogenesis of AKI in these patients may set the base for a more personalized approach, aimed at either preventing or reducing the potential harm associated with treatment.

The available data on AKI in patients with ischemic or hemorrhagic stroke are derived mostly from large administrative database where International Classification of Disease, 9th Revision (ICD-9) codes are used for diagnosis, dialysis identification and analysis of medical complications.

Notwithstanding a certain degree of variability in the accuracy of ICD-9 codes for the diagnosis of AKI and concomitant medical complications, the incidence of AKI in studies on patients with stroke ranges from 8 to $21 \%$, with higher figures being reported for intracranial hemorrhage (ICH), namely the less frequent but most severe form

Enrico Fiaccadori

enrico.fiaccadori@unipr.it

1 Dipartimento di Medicina e Chirurgia and Scuola di Specializzazione in Nefrologia, Università di Parma, Via Gramsci 14, 43100 Parma, Italy of stroke [5-7]. Moreover, literature data suggest that the incidence of AKI requiring dialysis in hospitalized patients with cerebrovascular accidents is growing, and confirm that AKI complicating stroke is associated with increased mortality and adverse post-discharge events in terms of long-term care facility need, disability, dependence [8].

The study by Wang and colleagues in the current issue of Internal and Emergency Medicine [9] confirms the high incidence of AKI among patients with stroke, but has some aspects of novelty. In fact, at variance with previous studies, the Authors evaluated a relatively large cohort of patients by applying for the first time the widely accepted 2012 KDIGO criteria, and assessed stroke severity by the National Institutes of Health Stroke Scale (NIHSS) score. Based on the KDIGO classification, a total of 135/647 (20.9\%) patients developed AKI in the neurology ICU, with 25/647 (3.8\%) reaching stage 3 AKI. Higher NIHSS score, lower baseline eGFR, previous hypertension, and ICU-acquired infectious complications (especially pneumonia) were demonstrated as significant risk factors for AKI; this latter was strongly associated with all-cause ICU mortality, and increasing severity of AKI (i.e., higher AKI stages) clearly paralleled death risk [9].

Although this study adds some interesting data to the topic, some limitations are to be acknowledged. First of all, it is a single-center study performed in a Chinese referral neurologic ICU. Most of the patients of the cohort (71.9\%) had hemorrhagic stroke, namely the most deadly form of stroke, while only a minority suffered from ischemic stroke. This is clearly at variance with what is commonly seen in clinical practice, and explains partially the high incidence of AKI, the unusually high mortality of patients with AKI complicating stroke (36.3\%), and also the high prevalence of some comorbidities (for example, $65.5 \%$ of the patients were hypertensive). Second, patients with 
reduced renal function (baseline eGFR $<60 \mathrm{ml} / \mathrm{min} /$ $1.73 \mathrm{~m}^{2}$ ) were thrice as frequent among AKI developers compared to the rest of the cohort. Pre-existing CKD is a frequent finding among stroke patients [10], and this very fact is reasonable, given the high cardiovascular disease burden intrinsic to CKD per se. CKD stage 2 or higher is a well known major risk factor for cardiovascular diseases including stroke [11], and bears a worse post-stroke prognosis [12]; however, the causes of CKD are not fully ascertained based on the data presented by Wang et al. [9]. Third, the causes of AKI in their patients were not completely defined. Patients with stroke are likely to be more prone to some specific forms of AKI, for example the one caused by mannitol toxicity [13]. Moreover, the potential toxicity of iodinated contrast media used for diagnostic, interventional and monitoring purposes, with the ensuing problem of radiocontrast-induced nephropathy, is to be underscored, although this issue is still highly debated [10, 14-16].

Finally, no data were presented on the incidence of AKI requiring renal replacement therapy (RRT), nor were the preferential RRT modalities or the anticoagulation protocols described. As a matter of fact, among the many unresolved issues concerning AKI in patients with stroke, the choice of both RRT modality and the anticoagulant agent for the extracorporeal circulation are likely to play a prominent role.

Literature data have in fact raised the potential negative impact of conventional intermittent hemodialysis on cerebral hemodynamics, especially in patients with AKI and higher risk for the loss of cerebral autoregulation and the development of the dialysis disequilibrium syndrome $[17,18]$. Even though few data are currently available on this topic, some AKI Guidelines suggest the preferential use of continuous modalities of RRT in the clinical setting of acute cerebral injury, especially in patients with high risk for endocranic hypertension [19]. As a matter of fact, it is intuitive that "softer" RRT modalities should be chosen, to avoid intradialytic hemodynamic instability and the risk of cerebral hypoperfusion.

For many years the activation of hemostasis in the extracorporeal circulation has represented the Achille's heel of RRT, especially in patients with AKI. No completely satisfying protocols had been established up to the recent implementation of the use of citrate as an anticoagulant agent in this clinical setting. In fact, citrate now represents the gold standard for RRT anticoagulation in AKI, since it allows true regional anticoagulation, i.e., a selective block of the whole hemostatic cascade only in the circuit and not in the patient's blood circulation [20]. This trivalent anion, which is mainly metabolized by the liver, inactivates many enzymatic reactions in the coagulation cascade by chelating ionized calcium, also blunting the activation of platelets and leucocytes in the circuit. Excellent circuit duration has been demonstrated with the use of citrate both in continuous (CRRT) [21] and prolonged intermittent (SLED) modalities of RRT [22], along with a reduced incidence of hemorrhagic complications. On these grounds, citrate is increasingly used in patients at high hemorrhagic risk, even when liver function is reduced, without any reported increase in hemorrhagic complication rates. However, no data are currently available about the safety and efficacy of citrate in patients with stroke, AKI and need for RRT.

In conclusion, further data on the epidemiology and pathogenesis of AKI in patients with stroke are needed, and papers like that of Wang and colleagues [8] are welcome. The availability of more detailed information on this relevant topic could foster our ability to more precisely tailor preventive strategies, as well as to better personalize RRT when needed.

\section{Compliance with ethical standards}

Conflict of interest The Authors declare that they have no conflict of interest.

Statements on human and animal rights This article does not contain any studies with human participants or animals performed by any of the authors.

Informed consent None.

\section{References}

1. Li PK, Burdmann EA, Mehta RL (2013) Acute kidney injury: global health alert. Kidney Int 83:372-376

2. Lameire NH, Bagga A, Cruz D et al (2013) Acute kidney injury: an increasing global concern. Lancet 382:170-179

3. Susantitaphong T, Cruz DN, Cerda J et al (2013) World incidence of AKI: a meta-analysis. Clin J Am Soc Nephrol 8:1482-1493

4. Odutayo A, Wong CX, Farkouh M et al (2017) AKI and longterm risk for cardiovascular events and mortality. Clin J Am Soc Nephrol 28:377-387

5. Khatri M, Himmelfarb J, Adams D, Becker K, Longstreth WT, Tirschwell DL (2014) Acute kidney injury is associated with increased hospital mortality after stroke. J Stroke Cerebrovasc Dis 23:25-30

6. Snarska K, Kapica-Topczewska K, Bachórzewska-Gajewska H, Małyszko J (2016) Renal function predicts outcomes in patients with ischaemic stroke and haemorrhagic stroke. Kidney Blood Press Res 41:424-433

7. Otite FO, Khandelwal P, Malik AM, Chaturvedi S, Sacco RL, Romano JG (2017) Ten-year temporal trends in medical complications after acute intracerebral hemorrhage in the United States. Stroke 48:596-603

8. Nadkarni GN, Patel AA, Konstantinidis J et al (2015) Dialysis requiring acute kidney injury in acute cerebrovascular accident hospitalizations. Stroke 46:3226-3231

9. Wang D, Guo Y, Zhang Y et al. (2017) Epidemiology of acute kidney injury in patients with stroke: a retrospective analysis from the neurology ICU. Int Emerg Med (in press) 
10. Demel SL, Grossman AW, Khoury JC et al (2017) Association between acute kidney disease and intravenous dye administration in patients with acute stroke: a population-based study. Stroke 48:835-839

11. Jurkovitz CT, Abramson JL, Vaccarino LV, Weintraub WS, McClellan WM (2003) Association of high serum creatinine and anemia increases the risk of coronary events: results from the prospective community-based atherosclerosis risk in communities (ARIC) study. J Am Soc Nephrol 14:2919-2925

12. El Husseini N, Fonarow GC, Smith EE et al (2017) Renal dysfunction is associated with poststroke discharge disposition and in-hospital mortality: findings from get with the guidelinesstroke. Stroke 48:327-334

13. Lin SY, Tang SC, Tsai LK et al (2015) Incidence and risk factors for acute kidney injury following mannitol infusion in patients with acute stroke: a retrospective cohort study. Medicine (Baltimore) 94:e2032-e2037

14. Ehrlich ME, Turner HL, Currie LJ, Wintermark M, Worrall BB, Southerland AM (2016) Safety of computed tomographic angiography in the evaluation of patients with acute stroke: a single-center experience stroke. Stroke 47:2045-2050

15. Tao Y, Dong W, Li Z, Chen Y et al (2017) Proteinuria as an independent risk factor for contrast-induced acute kidney injury and mortality in patients with stroke undergoing cerebral angiography. J Neurointerv Surg 9:445-448

16. Marchick MR, Allen BR, Weeks EC, Shuster JJ, Elie MC (2016) The incidence and significance of acute kidney injury following emergent contrast administration in patients with STEMI and stroke. Intern Emerg Med 11:853-857

17. Regolisti G, Maggiore U, Cademartiri C et al (2013) Cerebral blood flow decreases during intermittent hemodialysis in patients with acute kidney injury but not in patients with end-stage renal disease. Nephrology Dial Transplant 28:79-85

18. Regolisti G, Maggiore U et al (2013) Cerebral perfusion during intermittent hemodialysis in patients with acute kidney injury and advanced liver cirrhosis. J Nephrol 26:771-777

19. Kidney Disease: Improving Global Outcomes (KDIGO) Acute Kidney Injury Work Group. KDIGO Clinical Practice Guideline for Acute Kidney Injury (2012) Modality of renal replacement therapy for patients with AKI. Kidney Int Suppl 2:89-115

20. Morabito S, Tritapepe L, Pistolesi V, Fiaccadori E (2014) Regional citrate anticoagulation for renal replacement therapies in critically ill patients with acute kidney injury. Clin J Am Soc Nephrol 9:2173-2188

21. Liu C, Mao Z, Kang H, Hu J, Zhou F (2016) Regional citrate versus heparin anticoagulation for continuous renal replacement therapy in critically ill patients: a meta-analysis with trial sequential analysis of randomized controlled trials. Crit Care 20:144-156

22. Fiaccadori E, Regolisti G, Cademartiri C et al (2013) Efficacy and safety of a citrate-based protocol for sustained low-efficiency dialysis in AKI using standard dialysis equipment. Clin J Am Soc Nephrol 8:1670-1678 